Arq. Bras. Med. Vet. Zootec., v.66, n.4, p.1243-1250, 2014

\title{
Fósforo na alimentação de pacus criados em tanques-rede
}

\author{
[Phosphorus in the feeding of pacus reared in net-cages] \\ O. Diemer ${ }^{1}$, W.R. Boscolo ${ }^{2}$, A.A. Signor ${ }^{3}$, S. Klein ${ }^{4}$, A. Feiden ${ }^{2}$ \\ ${ }^{1}$ Aluno de pós-graduação - Universidade Estadual Paulista Júlio de Mesquita Filho - Unesp - Jaboticabal, SP \\ ${ }^{2}$ Universidade Estadual do Oeste do Paraná - Toledo, PR \\ ${ }^{3}$ Instituto Federal do Paraná - Foz do Iguaçu, PR \\ ${ }^{4}$ Aluno de pós-graduação - Universidade Estadual de Maringá - Maringá, PR
}

\begin{abstract}
RESUMO
Avaliaram-se o desempenho zootécnico, as características da carcaça, as características hematológicas e bioquímicas, a composição química e também a qualidade da água na criação de pacus Piaractus mesopotamicus em tanques-rede em reservatório e alimentados com dietas que continham diferentes quantidades de fósforo total. Foram utilizados 3.000 peixes, distribuídos em um delineamento inteiramente ao acaso, com cinco tratamentos e quatro repetições em 20 tanques-rede, sendo a unidade experimental composta por um tanque com 150 peixes. Os tratamentos foram constituídos por cinco dietas, elaboradas de modo a conterem quantidades de fósforo total de 6,0;7,5;9,0;10,5 e 12,0g/kg por meio da suplementação de fosfato bicálcico. A quantidade de 6,0 a $12,0 \mathrm{~g} / \mathrm{kg}$ de fósforo total na ração pode ser utilizada na alimentação de pacus criados em tanques-rede, sem que haja comprometimento do desempenho dos animais, e pode-se recomendar, comercialmente, $6,0 \mathrm{~g} / \mathrm{kg}$ de fósforo total em dietas para pacus.
\end{abstract}

Palavras-chave: características hematológicas, características bioquímicas, desempenho, peixe nativo

\begin{abstract}
The aim of this study was to evaluate the zootechnical performance, carcass characteristics, hematologic and biochemical characteristics, chemical composition and water quality in the rearing of pacus Piaractus mesopotamicus in cages in the reservoir fed with diets containing different total phosphorus quantities. A total of 3,000 fish allotted to a completely randomized design were used with five treatments and four replications in 20 cages, and the experimental unit consisted of a tank with 150 fish. The treatments were formed by five diets made to contain total phosphurus quantities of $6.0 ; 7.5 ; 9.0 ; 10.5$ and $12.0 \mathrm{~g} / \mathrm{kg}$ through supplementation of dicalcium phosphate. The quantities of 6.0 to $12.0 \mathrm{~g} / \mathrm{kg}$ of total phosphurus can be used in the feeding of pacus reared in cages without compromising the animals' performance and the use of $6.0 \mathrm{~g} / \mathrm{kg}$ total phosphorus in diets for pacus can be commercially recommended.
\end{abstract}

Keywords: Hematologic characteristics, biochemical characteristics, performance, native fish

\section{INTRODUÇÃO}

O pacu (Piaractus mesopotamicus), encontrado nas bacias dos rios Paraná, Paraguai e Uruguai, pertence à superordem Ostariophysi, cujos peixes são de alto valor comercial na pesca e na aquicultura brasileira. Apresenta hábito onívoro,

Recebido em 25 de junho de 2012

Aceito em 12 de dezembro de 2013

E-mail: odairdiemer@hotmail.com alimentando-se de folhas, sementes, caules, flores e frutos; porém, havendo a necessidade e a oportunidade, alimenta-se de insetos, aracnídeos, moluscos e peixes. É uma espécie promissora para aquicultura, pois apresenta rápido crescimento, rusticidade ao manejo, fácil adaptação à alimentação artificial e grande aceitação pelo mercado consumidor (Urbinati e Gonçalves, 2005). 
O Brasil tem potencial para aquicultura como poucos países do mundo em razão da quantidade de águas marítimas e continentais. No entanto, somente $1 \%$ dos corpos de água provenientes de barramento, lagos, lagoas, açudes, depósitos de águas pluviais e remansos de rios foi liberado para produção de pescado (Ostrensky et al., 2008). O aproveitamento dos recursos hídricos existentes tem proporcionado o desenvolvimento da criação de peixes em tanques-rede, que é uma alternativa de investimento de menor custo e maior rapidez de implantação, apontada como um agronegócio capaz de melhorar as condições sociais, ambientais e econômicas de uma região.

Os estudos relacionados às necessidades nutricionais dos peixes vêm se intensificando, principalmente com relação a fontes minerais que permitem melhor crescimento, rentabilidade econômica e menor impacto ambiental. Entre os minerais exigidos pelos peixes, o cálcio e o fósforo são requeridos em quantidades mais elevadas, quando comparados aos outros elementos minerais (Robinson et al., 1987). Contudo, os peixes podem absorver o fósforo e o cálcio da água por meio das brânquias, mas o fósforo em uma taxa muito menor que o cálcio (Hepher, 1988). Portanto, os peixes não obtêm o fósforo suficientemente do ambiente, assim é necessário suplementar as rações com esse elemento.

O fósforo destaca-se por ser de grande importância fisiológica e também por ser o principal agente eutrofizante do meio aquático. $\mathrm{O}$ presente estudo teve como objetivo avaliar o desempenho zootécnico, as características da carcaça, as características hematológicas e bioquímicas, a composição química e também a qualidade da água na criação de pacus em tanques-rede, alimentados com dietas que continham diferentes quantidades de fósforo total.

\section{MATERIAL E MÉTODOS}

O experimento foi realizado entre oito de fevereiro e três de maio de 2010, totalizando 85 dias experimentais, entre as coordenadas geográficas $\mathrm{W} 54^{\circ} 21^{\prime} 196, \mathrm{~S} 24^{\circ} 51^{\prime} 105$, W $54^{\circ}$ $21^{\prime} 078, \mathrm{~S} 24^{\circ} 51^{\prime} 192$ e W $54^{\circ} 21^{\prime} 224, \mathrm{~S} 24^{\circ} 51^{\prime}$ 143.
Foram utilizados 3000 peixes, com comprimento médio inicial de $17,85 \pm 0,57 \mathrm{~cm}$ e peso inicial médio de 115,23 $\pm 11,61 \mathrm{~g}$, distribuídos em um delineamento inteiramente ao acaso, com cinco tratamentos e quatro repetições, em 20 tanquesrede, com dimensões de 1,5 x 2,0 x 2,0m (comprimento $\mathrm{x}$ largura $\mathrm{x}$ profundidade), totalizando $5 \mathrm{~m}^{3}$ de volume útil, sendo a unidade experimental composta por um tanque com 150 peixes.

Os tratamentos foram constituídos por cinco dietas, elaboradas de modo a conterem quantidades de fósforo total de 6,$0 ; 7,5 ; 9,0 ; 10,5$ e $12,0 \mathrm{~g} / \mathrm{kg}$ por meio da suplementação de fosfato bicálcico. As dietas foram isoproteicas, isoenergéticas e isocálcicas e submetidas ao processamento de extrusão na fábrica-escola da Unioeste/GEMAq, localizada no município de Capitão Leônidas Marques - PR, e fornecidas três vezes ao dia, às nove, 14 e 17 horas, até a saciedade aparente (Tab. 1).

Para o monitoramento da qualidade da água, foram realizadas três coletas: no início, meio e fim do período experimental, sendo obtidas amostras de água de todos os tanques-rede. O oxigênio dissolvido, a temperatura da água, a condutividade elétrica, a turbidez e o $\mathrm{pH}$ foram avaliados in situ, com potenciômetros portáteis Hanna Instruments®.

As parcelas de água coletadas foram preservadas em garrafas de polietileno escuras e conservadas resfriadas para posterior análise. Determinaramse os parâmetros fósforo, ortofosfato, amônia e nitrito, seguindo a metodologia proposta por Mackerth et al. (1978) para fósforo e ortofosfato, Strickland e Parson (1972) para amônia e nitrito.

Ao final do período experimental, os animais permaneceram em jejum por 24 horas. Posteriormente, foram coletados quatro peixes de cada unidade experimental para a coleta de sangue. Para tanto, os animais foram anestesiados com Eugenol® (solução de óleo de cravo) $60 \mathrm{mg} . \mathrm{L}^{-1}$, e, em seguida, por punção caudal, foram coletados, com o auxílio de uma seringa descartável contendo EDTA (10\%), 2mL de sangue de cada animal. Essa alíquota foi destinada à contagem do número de eritrócitos em câmara de Neubauer, sob microscópio óptico com objetiva de 40 vezes, após a diluição do sangue com líquido de Hayem (Collier, 1944). 
As determinações de hemoglobina e hematócrito foram realizadas conforme métodos descritos por Collier (1944) e Goldenfarb et al. (1971), respectivamente. Com os valores obtidos da contagem de eritrócitos, hematócrito e taxa de hemoglobina, foram calculados os índices hematimétricos absolutos segundo Wintrobe (1934): volume corpuscular médio (VCM) e concentração de hemoglobina corpuscular média (CHCM).

Tabela 1. Composição alimentar e química na matéria natural das dietas experimentais com diferentes quantidades de fósforo total

\begin{tabular}{|c|c|c|c|c|c|}
\hline \multirow{2}{*}{ Ingredientes $(\mathrm{g} / \mathrm{kg})$} & \multicolumn{5}{|c|}{ Fósforo total $(\mathrm{g} / \mathrm{kg})$} \\
\hline & 6,0 & 7,5 & 9,0 & 10,5 & 12,0 \\
\hline Farelo de soja & 410 & 411 & 412 & 413,1 & 414,1 \\
\hline Milho grão & 333 & 328 & 322,6 & 317,6 & 311,9 \\
\hline Farelo de trigo & 150 & 150 & 150 & 150 & 150 \\
\hline Farinha vísceras aves & 29,8 & 30 & 30 & 30 & 30 \\
\hline Calcário & 27,8 & 22,6 & 17,3 & 12,0 & 6,8 \\
\hline Farinha de peixe & 20,0 & 20,0 & 20,0 & 20,0 & 20,0 \\
\hline Óleo de soja & 13,8 & 14,6 & 16,0 & 17,5 & 19,0 \\
\hline Premix* & 10,0 & 10,0 & 10,0 & 10,0 & 10,0 \\
\hline Sal & 3,0 & 3,0 & 3,0 & 3,0 & 3,0 \\
\hline L-Lisina & 1,4 & 1,4 & 1,4 & 1,4 & 1,4 \\
\hline Propionato & 1,0 & 1,0 & 1,0 & 1,0 & 1,0 \\
\hline BHT & 0,2 & 0,2 & 0,2 & 0,2 & 0,2 \\
\hline Fosfato bicálcico & - & 8,2 & 16,5 & 24,2 & 32,6 \\
\hline \multicolumn{6}{|c|}{ Nutrientes } \\
\hline Amido $(\mathrm{g} / \mathrm{kg})$ & 252 & 249 & 245,7 & 242,3 & 239,0 \\
\hline Cálcio (g/kg) & 15,0 & 15,0 & 15,0 & 15,0 & 15,0 \\
\hline Energia digestível $(\mathrm{kcal} / \mathrm{kg})$ & 3.000 & 3.000 & 3.000 & 3.000 & 3.000 \\
\hline Fósforo total (g/kg) & 6,0 & 7,5 & 9,0 & 10,5 & 12,0 \\
\hline Gordura $(\mathrm{g} / \mathrm{kg})$ & 37,8 & 39,0 & 40,3 & 41,6 & 42,8 \\
\hline Lisina total $(\mathrm{g} / \mathrm{kg})$ & 16,4 & 16,4 & 16,4 & 16,4 & 16,4 \\
\hline Metionina $(\mathrm{g} / \mathrm{kg})$ & 4,20 & 4,20 & 4,20 & 4,20 & 4,20 \\
\hline Proteína bruta $(\mathrm{g} / \mathrm{kg})$ & 270 & 270 & 270 & 270 & 27,00 \\
\hline
\end{tabular}

*Garantia por quilograma do produto: vit. A - 500.000UI; vit. D3 - 250.000UI; vit. E - 5.000mg; vit. K3 - 500mg; vit. B1 - 1.500mg; vit. B2 - 1.500mg; vit. B6 - 1.500mg; vit. B12 - 4.000mg; ác. fólico - 500mg; pantotenato Ca $4.000 \mathrm{mg}$; vit. C - $10.000 \mathrm{mg}$; biotina - 10mg; inositol - 1.000; nicotinamida - 7.000; colina - 10.000mg; Co - 10mg; $\mathrm{Cu}$ - 1.000mg; Fe - 5.000mg; I - 200mg; Mn - 1500mg; Se - 30mg; Zn - 9.000mg.

Para avaliação dos triglicerídeos e das proteínas do sangue mediante análises bioquímicas, foi coletado $1 \mathrm{~mL}$ de soro, sem anticoagulante. As determinações das concentrações foram realizadas utilizando-se kits específicos para cada análise (Gold Analisa $\left.{ }^{\circledR}\right)$, e a leitura foi feita em espectrofotometria com comprimento de onda específico para cada análise.

Posteriormente, todos os peixes dos tanques-rede foram coletados e anestesiados para a avaliação dos índices zootécnicos de peso final, ganho de peso, conversão alimentar, sobrevivência, taxa de crescimento específico (TCE) e fator de condição (FC). A taxa de crescimento específico foi determinada conforme a equação: $\mathrm{TCE}=[(\ln$ peso médio final $(\mathrm{g})$ - $\ln$ peso médio inicial (g))/tempo de experimento (dias)] x 100. O fator de condição foi obtido conforme a fórmula: $\mathrm{FC}=\left[\right.$ peso/(comprimento total $\left.\left.^{-3}\right) \times 100\right]$. Em seguida, foram retirados, aleatoriamente, oito animais, seis para avaliação das características da carcaça e dois para avaliação da composição química, de cada tanque-rede, insensibilizados por hipotermia, acondicionados em caixa com gelo e transportados ao laboratório, onde foram avaliados os seguintes índices de rendimento da carcaça: comprimento total, comprimento padrão, peso total, peso eviscerado, tronco limpo, filé, cabeça, gordura visceral e índice hepatossomático. Para isso, os animais foram pesados em balança digital, abertos na região ventral e eviscerados. Logo após, foram efetuados os cortes referentes à separação da cabeça, nadadeiras, couro e filé. As vísceras foram colocadas em placas de Petry, de onde se 
separou a gordura e o fígado; o material resultante foi pesado em balança analítica. Dois peixes de cada tanque-rede foram separados e moídos inteiros para análise da composição química segundo descrição da AOAC (2005). Avaliaram-se umidade (UM), proteína bruta $(\mathrm{PB})$, extrato etéreo (EE) e matéria mineral (MM).

Os dados referentes às características avaliadas foram tabulados e submetidos à análise de variância e à regressão, por meio do programa estatístico SAEG.

\section{RESULTADOS E DISCUSSÃO}

Os resultados de peso médio inicial, peso médio final, ganho de peso, conversão alimentar, taxa de crescimento específico e fator de condição não apresentaram diferença $(\mathrm{P}>0,05)$ entre tratamentos (Tab. 2). Ao final do período experimental, não foi verificada mortalidade em nenhum dos tratamentos.

Tabela 2. Desempenho de pacus (Piaractus mesopotamicus) criados em tanques-rede e alimentados com dietas que continham diferentes quantidade de fósforo total

\begin{tabular}{|c|c|c|c|c|c|c|}
\hline \multirow{2}{*}{ Características } & \multicolumn{5}{|c|}{ Fósforo total $(\mathrm{g} / \mathrm{kg})$} & \multirow{2}{*}{$\mathrm{CV}(\%)$} \\
\hline & 6,0 & 7,5 & 9,0 & 10,5 & 12,0 & \\
\hline Peso médio inicial $(\mathrm{g})$ & 118,33 & 108,33 & 115,66 & 121,50 & 114,83 & 10,42 \\
\hline Peso médio final $(\mathrm{g})$ & 383,57 & 374,56 & 358,53 & 384,82 & 383,73 & 4,82 \\
\hline Ganho de peso (g) & 265,24 & 266,22 & 242,86 & 263,32 & 269,40 & 8,44 \\
\hline Conversão alimentar & 2,67 & 2,69 & 2,91 & 2,69 & 2,62 & 8,87 \\
\hline Taxa crescimento específico & 1,47 & 1,55 & 1,41 & 1,44 & 1,51 & 10,03 \\
\hline Fator de condição & 2,50 & 2,54 & 2,43 & 2,40 & 2,74 & 9,41 \\
\hline
\end{tabular}

Valores obtidos segundo os tratamentos não diferem entre si ( $p>0,05)$.

O pacu apresentou excelente aproveitamento do fósforo contido nos ingredientes utilizados para a elaboração das rações. De acordo com DiasKoberstein et al. (2005), o pacu, em ambiente natural, alimenta-se basicamente de folhas e de frutas de árvores, o que leva a crer que esse peixe possui habilidade especial na digestão e na absorção de alimentos. Silva (1985) observou que essa espécie apresenta adaptações alternativas de sua alimentação não só na composição mas também no grau de fracionamento dos alimentos encontrados no seu trato digestório, assim consegue maximizar a digestão e a absorção e, consequentemente, extrair do alimento o máximo de nutrientes.

Segundo Pezzato et al. (2006), para alevinos de tilápia-do-nilo é necessária a quantidade mínima de $7,5 \mathrm{~g} / \mathrm{kg}$ de fósforo disponível na dieta, enquanto Furuya et al. (2008a) determinaram a exigência de fósforo disponível em $5,2 \mathrm{~g} / \mathrm{kg}$ para juvenis de tilápia-do-nilo. De acordo com os autores, o ganho de peso foi maior nos peixes alimentados com quantidades mais elevadas de fósforo, condições diferentes da observada no presente estudo com juvenis de pacu, o que sugere que esta espécie apresenta menor exigência de fósforo que a tilápia.
Os resultados obtidos demonstraram que não foi necessária a suplementação de fósforo por meio de fosfato bicálcico em dietas formuladas com ingredientes convencionais e que contenham $6,0 \mathrm{~g} / \mathrm{kg}$ de fósforo total. Signor et al. (2011), ao estudarem o fósforo na alimentação de pacu, afirmaram que as quantidades de fósforo usadas por eles não influenciaram o desempenho produtivo, o rendimento e a composição química e que $4,0 \mathrm{~g} / \mathrm{kg}$ de fósforo total atendem às exigências dos peixes. Esse valor é menor que o valor mais baixo utilizado neste trabalho.

Furuya et al. (2008b), ao avaliarem a adição de fitase em rações para juvenis de pacu, observaram aumento linear do efeito da fitase nas dietas sobre o ganho de peso e cinzas na carcaça e nos ossos, efeito quadrático sobre a conversão alimentar e taxa de eficiência proteica sobre a quantidade de cálcio e fósforo nos ossos. De maneira geral, os efeitos positivos da adição de fitase sobre o desempenho produtivo e a retenção de minerais na carcaça possivelmente estão relacionados com a maior disponibilidade do fósforo.

Os resultados das características da carcaça não apresentaram diferenças $(\mathrm{P}>0,05)$ entre os diferentes tratamentos (Tab. 3). 
Os rendimentos de carcaça e tronco limpo foram semelhantes aos encontrados por Bombardelli et al. (2007) para o pacu. Esses autores obtiveram 84,40 e $57,94 \mathrm{~kg} / 100 \mathrm{~kg}$, respectivamente. Os valores de tronco limpo foram mais altos que os encontrados por Boscolo et al. (2001), que avaliaram o rendimento de duas linhagens de tilápia-do-nilo: tailandesa e comum, 49,46 e $51,39 \mathrm{~kg} / 100 \mathrm{~kg}$, respectivamente.

Tabela 3. Características da carcaça de pacus (Piaractus mesopotamicus) criados em tanques-rede e alimentados com dietas que continham diferentes quantidades de fósforo total

\begin{tabular}{|c|c|c|c|c|c|c|}
\hline \multirow{2}{*}{ Características } & \multicolumn{5}{|c|}{ Fósforo total $(\mathrm{g} / \mathrm{kg})$} & \multirow{2}{*}{$\mathrm{CV}(\%)$} \\
\hline & 6,0 & 7,5 & 9,0 & 10,5 & 12,0 & \\
\hline Comprimento total $(\mathrm{cm})$ & 25,16 & 24,37 & 25,75 & 25,87 & 24,25 & 7,35 \\
\hline Comprimento padrão $(\mathrm{cm})$ & 21,66 & 20,85 & 22,37 & 22,25 & 21,00 & 6,94 \\
\hline Rendimento de carcaça $(\mathrm{kg} / 100 \mathrm{~kg})$ & 87,51 & 87,25 & 85,44 & 85,71 & 86,63 & 2,29 \\
\hline Tronco limpo (kg/100kg ) & 52,00 & 56,80 & 52,60 & 55,04 & 54,01 & 7,11 \\
\hline Filé $(\mathrm{kg} / 100 \mathrm{~kg})$ & 35,10 & 38,57 & 40,58 & 38,21 & 36,68 & 11,57 \\
\hline Cabeça (kg/100kg) & 21,11 & 19,56 & 21,88 & 19,63 & 19,80 & 10,70 \\
\hline Gordura visceral $(\mathrm{kg} / 100 \mathrm{~kg})$ & 5,59 & 6,00 & 5,25 & 5,27 & 5,14 & 23,41 \\
\hline Índice hepatossomático & 0,57 & 0,48 & 0,45 & 0,51 & 0,58 & 33,87 \\
\hline
\end{tabular}

Valores obtidos segundo os tratamentos não diferem entre si $(p>0,05)$.

Rendimento, tronco limpo e gordura visceral foram semelhantes aos encontrados por Hilbig (2010) para a mesma espécie, cujos valores foram $88,70,54,38$ e $5,96 \mathrm{~kg} / 100 \mathrm{~kg}$, respectivamente, enquanto para o filé e o índice hepatossomático foram diferentes. O valor de filé é semelhante ao relatado por Gomiero et al. (2003), quando avaliaram o rendimento de carcaça de matrinxã (Brycon cephalus) nos diferentes cortes de cabeça.

Não se observaram diferenças $(\mathrm{P}>0,05)$ quanto às características hematológicas e bioquímicas nos distintos tratamentos (Tab. 4), o que sugere que todos os animais estavam em bom estado de homeostase e ainda permaneceram dentro dos padrões para a espécie segundo Tavares-Dias e Moraes (2004).

Os resultados de hemoglobina, hematócrino e eritrócito corroboraram os obtidos por Bittencourt et al. (2010) para a mesma espécie. Esses autores encontraram 9,98g.dL $\mathrm{dL}^{-1}, 42,93 \% \mathrm{e}$ $1,8810^{6} \mu \mathrm{L}$, respectivamente.

Chagas e Val (2003), ao avaliarem variáveis hematológicos de tambaqui (Colossoma macropomum), verificaram que a redução no hematócrito caracterizou anemia nos peixes, o que não foi observado nos peixes deste experimento.

Tabela 4. Características hematológicas e bioquímicas de pacus (Piaractus mesopotamicus) criados em tanques-rede e alimentados com dietas que continham diferentes quantidades de fósforo total

\begin{tabular}{|c|c|c|c|c|c|c|}
\hline \multirow{2}{*}{ Características } & \multicolumn{5}{|c|}{ Fósforo total $(\mathrm{g} / \mathrm{kg})$} & \multirow{2}{*}{$\mathrm{CV}(\%)$} \\
\hline & 6,0 & 7,5 & 9,0 & 10,5 & 12,0 & \\
\hline Hemoglobina $\left(\mathrm{g} . \mathrm{dL}^{-1}\right)$ & 10,83 & 10,33 & 10,93 & 10,11 & 10,57 & 5,87 \\
\hline Hematócrino (\%) & 34,00 & 31,50 & 33,77 & 33,33 & 32,66 & 3,13 \\
\hline Eritrócito $\left(10^{6} \mu \mathrm{L}\right)$ & 1,80 & 1,72 & 1,73 & 1,77 & 1,80 & 4,33 \\
\hline Triglicerídios $\left(\mathrm{mg} \cdot \mathrm{dL}^{-1}\right)$ & 261,97 & 216,32 & 255,44 & 250,13 & 298,43 & 26,89 \\
\hline Proteínas $\left(\mathrm{g} \cdot \mathrm{dL}^{-1}\right)$ & 3,63 & 3,71 & 3,44 & 3,54 & 3,37 & 13,54 \\
\hline $\mathrm{VCM}$ & 188,57 & 183,21 & 195,27 & 187,86 & 181,50 & 4,73 \\
\hline $\mathrm{HCM}$ & 60,15 & 60,07 & 63,25 & 57,03 & 58,68 & 7,08 \\
\hline CHCM & 3,19 & 3,28 & 3,23 & 3,03 & 3,24 & 6,57 \\
\hline
\end{tabular}

Valores obtidos segundo os tratamentos não diferem entre si $(\mathrm{P}>0,05)$.

Os resultados de qualidade de água não apresentaram efeito $(\mathrm{P}>0,05)$ das diferentes quantidades de fósforo total (Tab. 5).
A lixiviação do fósforo total contido na ração não comprometeu a qualidade da água de criação de pacus em tanques-rede, já que o ambiente utilizado para o estudo foi aberto, o que 
possibilitou a circulação total da água. Provavelmente, se o experimento fosse realizado em ambiente fechado, sem renovação de água, as características desta seriam alteradas, aspectos já relatado por Diemer et al. (2010a), ao verificarem a qualidade da água após a alimentação de jundiás (Rhamdia voulezi) com dietas com diferentes quantidades de fósforo total.

Tabela 5. Valores dos parâmetros abióticos analisados na criação de pacus (Piaractus mesopotamicus) alimentados com rações contendo diferentes quantidades de fósforo total

\begin{tabular}{|c|c|c|c|c|c|c|}
\hline \multirow{2}{*}{ Parâmetro } & \multicolumn{5}{|c|}{ Fósforo total $(\mathrm{g} / \mathrm{kg})$} & \multirow{2}{*}{$\begin{array}{l}\text { CV } \\
(\%)\end{array}$} \\
\hline & 60 & 75 & 90 & 105 & 120 & \\
\hline Turbidez (NTU) & 8,05 & 7,68 & 7,70 & 7,95 & 7,97 & 2,55 \\
\hline $\mathrm{pH}$ & 7,50 & 7,56 & 7,58 & 7,53 & 7,59 & 0,87 \\
\hline Condutividade elétrica $\left(\mu \mathrm{s} . \mathrm{cm}^{-1}\right)$ & 40,00 & 46,68 & 40,00 & 40,00 & 46,67 & 8,55 \\
\hline Oxigênio $\left(\mathrm{mg} \cdot \mathrm{L}^{-1}\right)$ & 5,87 & 5,99 & 5,80 & 5,83 & 5,77 & 2,03 \\
\hline Temperatura $\left({ }^{\circ} \mathrm{C}\right)$ & 30,57 & 30,67 & 30,57 & 30,50 & 30,67 & 0,91 \\
\hline Amônia $\left(m g . L^{-1}\right)$ & 0,072 & 0,048 & 0,081 & 0,061 & 0,093 & 32,0 \\
\hline Ortofosfato $\left(\mathrm{mg} . \mathrm{L}^{-1}\right)$ & 0,022 & 0,018 & 0,016 & 0,014 & 0,049 & 76,9 \\
\hline Nitrito (mg.L $\left.{ }^{-1}\right)$ & 0,008 & 0,009 & 0,008 & 0,008 & 0,009 & 16,8 \\
\hline Fósforo (mg. $\left.\mathrm{L}^{-1}\right)$ & 0,058 & 0,040 & 0,050 & 0,037 & 0,090 & 48,8 \\
\hline
\end{tabular}

Valores obtidos segundo os tratamentos não diferem entre si $(\mathrm{P}>0,05)$.

Os parâmetros de qualidade de água foram próximos aos resultados apresentados por Diemer et al. (2010b), para o mesmo reservatório, quando analisaram a dinâmica nictimeral e vertical das características limnológicas em ambiente de criação de peixes nativos em tanques-rede. Esses autores relataram também que o reservatório apresenta características propícias para o cultivo de peixes em tanques-rede.
Os resultados da composição centesimal (Fig. 1) são similares aos encontrados por Bittencourt (2010), quando analisou a composição da carcaça no cultivo de pacu sob diferentes densidades em tanque-rede no mesmo reservatório. A composição química do pacu encontrado neste trabalho corrobora os estudos de Arbeláez-Rojas et al. (2002) ao avaliarem a composição corporal de tambaqui em sistemas de cultivo intensivo e semi-intensivo.

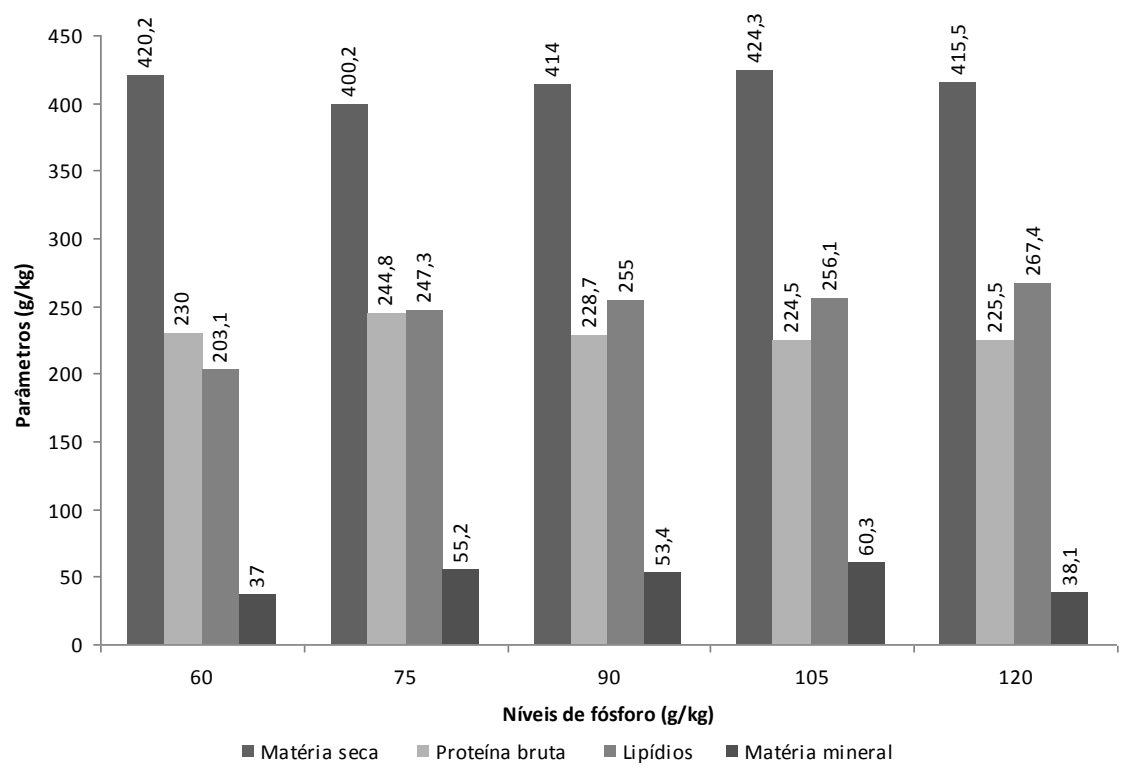

Figura 1. Composição química da carcaça na matéria natural de pacus (Piaractus mesopotamicus) criados em tanques-rede e alimentados com dietas que continham diferentes quantidades de fósforo total. 


\section{CONCLUSÕES}

A quantidade de 6,0 a $12,0 \mathrm{~g} / \mathrm{kg}$ de fósforo total na ração pode ser utilizada na alimentação de pacus criados em tanques-rede, sem que haja comprometimento do desempenho dos animais. No entanto, é fundamental destacar a importância do fósforo para os cálculos de capacidade de suporte de reservatórios com cultivo em tanques-rede, pois esse mineral é um potencial poluidor dos ambientes aquáticos. Assim, pode-se recomendar, comercialmente, a utilização de $6,0 \mathrm{~g} / \mathrm{kg}$ de fósforo total na alimentação de pacus criados em tanques-rede, quantidade que irá proporcionar bom desempenho dos peixes.

\section{REFERÊNCIAS}

ARBELÁEZ-ROJAS, G.A.; FRACALOSSI, D.M.; FIM, J.D.I. Composição corporal de tambaqui (Colossoma macropomum) e matrinxã (Brycon cephalus) em sistemas de cultivo intensivo e em igarapé semi-intensivo em viveiros. Rev. Bras. Zootec., v.31, p.1059-1069, 2002.

ASSOCIATION OF OFFICIAL ANALYTICAL CHEMISTS. Oficial Methods of Analysis. 17, ed. Arlington: v.2, 2005.

BITTENCOURT, F.; FEIDEN, A.; SIGNOR, A.A. et al. Densidade de estocagem e parâmetros eritrocitários de pacus criados em tanques-rede. Rev. Bras. Zootec., v.39, p.2323-2329, 2010.

BOMBARDELLI, R.A.; BENCKE B.C.; SANCHES, E.A. Processamento da carne do pacu (Piaractus mesopotamicus) cultivado em tanques-rede no reservatório de Itaipu. Acta Scienti. Anim. Sci., v.29, p.457-463, 2007.

BOSCOLO, W.R.; HAYASHI, C.; SOARES, C.M. et al. Desempenho e características de carcaça de machos revertidos de tilápias do Nilo (Oreochromis niloticus), linhagens tailandesa e comum, nas fases inicial e de crescimento. Rev. Bras. Zootec., v.30, p.1391-1396, 2001.

CHAGAS, E.C.; VAL, A.L. Efeito da vitamina $\mathrm{C}$ no ganho de peso e em parâmetros hematológicos de tambaqui (Colossoma macropomum). Pesqu. Agrop. Bras., v.38, p.397402, 2003.
COLLIER, H.B. The standardizations of blood haemoglobin determinations. Canadian Med. Associat. J., v.50, p.550-552, 1944.

DIAS-KOBERSTEIN, T.C.R.; CARNEIRO, D.J.; URBINATI, E.C. Tempo de trânsito gastrintestinal e esvaziamento gástrico do pacu (Piaractus mesopotamicus) em diferentes temperaturas de cultivo. Acta Sci. Anim. Sci., v.27, p.413-417, 2005.

DIEMER, O.; BOSCOLO, W.R.; DIETERICH, T.G. et al. Rações contendo diferentes níveis de fósforo total na alimentação de jundiás (Rhamdia voulezi): qualidade da água. In: II SIMPÓSIO NACIONAL DE ENGENHARIA DE PESCA, 2., 2010, Paraná. Anais... , Paraná: 2010a.

DIEMER, O.; NEU, D.H.; FEIDEN, A. et al. Dinâmica nictimeral e vertical das características limnológicas em ambiente de criação de peixes nativos em tanques-rede. Cienc. Anim. Bras., v.11, p.24-31, 2010b.

FURUYA, W.M.; FUGII, K.M.; SANTOS, L.D. et al. Exigência de fósforo disponível para juvenis de tilápia do Nilo. Rev. Bras. Zootec., v.37, p.1517-1522, 2008a.

FURUYA, W.M.; MICHELATO, M.; SILVA, L.C.R. et al. Fitase em rações para juvenis de pacu (Piaractus mesopotamicus). Bol. Instit. da Pesca, v.34, p.489-496, 2008b.

GOMIERO, J.S.G.; RIBEIRO, P.A.P. FERREIRA, M.W. et al. Rendimento de carcaça de peixe matrinxã (Brycon cephalus) nos diferentes cortes de cabeça. Cienc. Agrotec., v.27, p.211-216, 2003.

GOLDENFARB, P.B.; BOWYER, F.P.; HALL, E.; BROSIUS, E. Reproducibility in the hematology laboratory: the microhematocrit determinations. Am. J. Clin. Pathol., v.56, p.3539, 1971.

HEPHER, B. Nutrition of Pond Fishes. New York: CAMBRIDGE UNIVERSITY PRESS, 1988. 388p.

HILBIG, C.C. Taxa de arraçoamento para o pacu (Piaractus mesopotamicus) criados em tanques-rede no reservatório de Itaipu. Dissertação (Mestrado em Zootecnia). Universidade Estadual do Oeste do Paraná. 46p. 2010. 
MACKERETH, J.F.H.; HERON, J.; TALLING, J.F. Water analysis: some revised methods for limnologists. Freshwater Biol. Assoc., v.36, p.121, 1978.

OSTRENSKY, A.; BORGHETTI, J.R.; SOTO, D. Aquicultura no Brasil: o desafio é crescer. Brasília-DF, 2008. 276 p.

PEZZATO, L.E.; ROSA, M.J.S.; BARROS, M.M. et al. Exigência em fósforo disponível para alevinos de tilápia do Nilo. Cienc. Rural, v.36, p.1600-1605, 2006.

ROBINSON, E.H.; LABOMASCUS, D.; BROWN, P.B. et al. Dietary calcium and phosphorus requirements of Oreochromis aureus reared in calcium-free water. Aquaculture, v.64, p.267-276, 1987.

SAEG - UNIVERSIDADE FEDERAL DE VIÇOSA. UFV. Sistema para análises estatísticas e genéticas. Versão 7.1. Viçosa, MG. 150p. 1997.
SIGNOR, A.A.; FEIDEN, A.; BITTENCOURT, F. et al. Fósforo na alimentação de pacu (Piaractus mesopotamicus). Rev. Bras. Zootec., v.40, p.2646-2650, 2011.

SILVA, A.J. Aspectos da alimentação do pacu adulto, Colossoma mitrei (BERG,1895) (Pisces, Characidae), no Pantanal de Mato Grosso. Dissertação (Mestrado em Ciências Biológicas/Zoologia) - Universidade Federal do Rio de Janeiro, Rio de Janeiro, 1985.

STRICKLAND, J.D.H.; PARSONS, T.R. A pratical handbook of sea water analysis. Ottawa: Fish Res. Board of Canada. 1972. 310p.

TAVARES-DIAS, M.; MORAES, F.R. Hematologia de peixes teleósteos. Ribeirão Preto: Villimpress Complexo Gráfico, 144p. 2004.

URBINATI, E.C.; GONÇALVES, F.D. Pacu (Piaractus mesopotamicus). In: BALDISSEROTO, B.; GOMES, L.C. Espécies nativas para piscicultura no Brasil. Santa Maria: Editora UFSM, 2005. 470p. 